\title{
Valoración diagnóstica de enzimas hepáticas en perfiles bioquímicos sanguíneos de vacas lecheras
}

\section{Diagnostic assessment of liver enzymes in blood profiles from dairy cows}

\author{
Mirela Noro, ${ }^{1 *}$ Ph.D, Pía Cid T, ${ }^{2}$ Esp, Catalina Wagemann F, ${ }^{2}$ M.Sc, Verónica Arnés $V_{,}^{2}$ Tec Med, \\ Fernando Wittwer $\mathrm{M}^{2}{ }^{2}$ M.Sc.
}

\begin{abstract}
${ }^{1}$ Universidade Federal do Pampa, UNIPAMPA, Curso Medicina Veterinaria. Uruguaiana, Brasil. 2Universidad Austral de Chile, UACh, Instituto Ciencias Clínicas Veterinarias. Casilla 567, Valdivia, Chile. Correspondencia: mirelanoro@gmail.com
\end{abstract}

Recibido: Marzo de 2012; Aceptado: Diciembre de 2012.

\section{RESUMEN}

Objetivo. El objetivo del estudio fue determinar la asociación, sensibilidad (Se) y especificidad (Es) de las enzimas glutamato deshidrogenasa (GDH, EC: 1.4.1.3), aspartato aminotransferasa (AST, EC: 2.6.1.1) y g-glutamil transpeptidasa (GGT, EC: 2.3.2.2) como indicadores de daño hepático en perfiles bioquímicos sanguíneos de vacas lecheras. Materiales y métodos. Se analizaron los valores de la actividad plasmática de GDH, AST y GGT, obtenidos de 1566 vacas correspondientes a 112 perfiles bioquímicos realizados en rebaños lecheros. Se determinó la asociación entre la actividad de las enzimas y el área bajo la curva $(A B C)$ y la Se y la Es mediante curva ROC considerando la presencia de daño hepático cuando GDH $>30 \mathrm{U} / \mathrm{L}$ o GGT > $39 \mathrm{U} / \mathrm{L}$. Resultados. La AST presentó mayor asociación con la GDH $(r=0.55)$ que la GGT $(r=0.44)$, así como una mayor $A B C(p=0.01)$ al utilizar GDH $>30 \mathrm{U} / \mathrm{L}$ para indicar daño hepático. El grado de asociación entre AST y GGT fue de $r=0.42$. La sensibilidad de la AST para detectar daño hepático, utilizando el punto de corte de referencia del laboratorio ( $>150$ $\mathrm{U} / \mathrm{L}$ ), fue inferior que al emplear GGT (>39 U/L). El punto crítico de máxima Se y Es de la curva ROC para daño hepático se obtuvo con AST>110 U/L, valor que incrementó la concordancia diagnóstica con la GDH (Kappa >0.300), pero no con la GGT. Conclusiones. La actividad plasmática de AST en vacas lecheras se asocia mayormente con la de GDH que con la de GGT y su límite de referencia para predecir daño hepático correspondería a >110 U/L.

Palabras clave: Enzimas hepáticas, perfiles sanguíneos, vacas lecheras (Fuente: $C A B$ ). 


\section{ABSTRACT}

Objective. The aim of this study was to establish the association, sensibility (Se) and specificity (Sp) of the plasmatic activity of glutamate dehydrogenase (GDH, EC: 1.4.1.3), aspartate aminotransferase (AST, EC: 2.6.1.1) and g-glutamil transpeptidase (GGT, EC: 2.3.2.2) as liver damage markers in blood profiles of dairy cows. Materials and methods. The records of the plasma activity for GDH, AST and GGT obtained from blood profiles taken from 1566 cows from 112 dairy herds. The association between enzymatic activity, area below the curve $(A B C)$, and Se and Sp using a ROC curve were determined, considering liver damage when GDH $>30 \mathrm{U} / \mathrm{L}$ or GGT >39 U/L. Results. The AST was more likely associated to GDH $(r=0.55)$ than to GGT $(r=0.44)$. AST also had a higher ABC $(p=0.01)$ when using GDH $>30 \mathrm{U} / \mathrm{L}$ as an indicator of liver damage. The correlation between AST and GGT was of $r=0.42$. AST Se to detect liver damage with a cut-off level of $>150 \mathrm{U} / \mathrm{L}$ was lower than the Se of GGT. The critical point for maximum Se and Sp of the ROC curve was obtained with AST>110 U/L and an increase in the diagnostic concordance with GDH (Kappa>0.300), was obtained using this value. Conclusions. Plasma activity of AST is more associated to GDH than to GGT in dairy cows. The cut-off level of plasma activity of AST for liver damage prediction in dairy cows would be $>110 \mathrm{U} / \mathrm{L}$.

Key words: Dairy cow, liver enzymes, blood profile (Source: $C A B$ ).

\section{INTRODUCCIÓN}

El hígado es un órgano vital para el metabolismo al ser responsable de la gluconeogénesis, ureagénesis, metabolismo de lípidos y procesos de detoxificación (1). En los sistemas de producción láctea la vaca debe sostener una alta exigencia productiva donde el hígado cumple un rol metabólico fundamental, estando sujeto a la presentación de trastornos metabólicos (2) y tóxicos (3); consecuentemente para mantener el estatus sanitario y productivo del rebaño, es fundamental monitorear su integridad en los programas de salud y nutrición.

El examen directo del hígado no es realizable en rumiantes siendo necesario recurrir a pruebas complementarias para detectar lesiones o disfunciones discretas, cuya magnitud no culmina en una alteración clínica. Ningún analito es específico de enfermedad hepática, si bien diversos constituyentes sanguíneos son modificados cuantitativamente en alteraciones del hígado. Las enzimas hepáticas están presentes en los hepatocitos o en el epitelio de los canalículos biliares, de modo que su lesión condiciona un aumento en la circulación sanguínea; por ello, el incremento de su actividad plasmática es indicativo de pérdida en la integridad celular hepática o de colestasis (4).

Entre las enzimas hepáticas utilizadas en el diagnóstico clínico de enfermedades hepatocelulares o colestasis en rumiantes están la glutamato deshidrogenasa (GDH, EC: 1.4.1.3), la aspartato amino transferasa (AST, EC: 2.6.1.1) y la g-glutamil transpeptidasa (GGT, EC: 2.3 .2 .2 ), las cuales permiten diagnosticar la presencia de daño hepático y orientar la ubicación de la lesión $(1,5)$. La GDH y GGT se consideran órgano-específicas, la primera es hepatocelular y la segunda canalicular, mientras que la AST siendo hepatocelular también se ubica en células musculares y otras $(5,6)$.

En el sur de Chile la presentación de trastornos hepáticos, diagnosticada mediante el aumento de la actividad plasmática de las enzimas AST y GGT, incrementó en el período entre los años de 2003 a 2010 comparado con 1986 a 2002 (7), resultado atribuible a una intensificación en los sistemas productivos con presentación de trastornos metabólicos como la lipidosis hepática (2) y el uso de alimentos conservados contaminados con micotoxinas (3). Por otro lado, en los exámenes bioquímicos los valores de las diferentes enzimas no siempre se asocian en cuadros de alteración hepática, situación que dificulta la interpretación de los resultados. Este hecho se atribuye a las diferencias en la ubicación de las enzimas, la etiología, severidad y curso del daño hepático, así como a los intervalos de referencia utilizados. Para comparar diferentes pruebas diagnósticas, así como su exactitud, se determina el área bajo la curva ROC $(A B C)$ y se estima su sensibilidad (Se) y especificidad (Es) (8), es así que disponer de datos de asociación y de sensibilidad entre pruebas diagnósticas es imprescindible para un diagnóstico certero y ajuste de los intervalos de referencia.

El objetivo del estudio fue determinar la asociación, la Se y la Es de las enzimas GDH, AST y GGT como indicadores de daño hepático en perfiles bioquímicos sanguíneos de vacas lecheras. 


\section{MATERIALES Y MÉTODOS}

Fuentes de información. Se analizaron los valores de la actividad plasmática de las enzimas hepáticas GDH, AST y GGT obtenidos de 1566 vacas correspondientes a 112 perfiles bioquímicos sanguíneos realizados a rebaños lecheros ubicados entre las regiones del Bío Bío y Los Lagos, Chile. Cada perfil estuvo constituido por un grupo de 7 a 28 vacas lecheras; en ellos estuvieron disponibles los valores de dos o de las tres enzimas evaluadas en el estudio. Los análisis fueron realizados en el Laboratorio de Patología Clínica Veterinaria de la Universidad Austral de Chile entre enero de 2008 y junio de 2011.

Análisis de laboratorio. La actividad de GDH (Diasys $^{\circledR}$, Ref. 124119910021), AST (Human ${ }^{\circledR}$, Ref. 12021) y GGT (Human ${ }^{\circledR}$, Ref. 12013) se determinó en muestras de plasma heparinizado en un autoanalizador Metrolab $2300^{\circledR}$ (Wiener Lab) a $37^{\circ} \mathrm{C}$.

Análisis estadístico. Se utilizaron modelos univariados considerando que no todos los perfiles contaban con los datos de las 3 enzimas. La asociación entre las actividades enzimáticas fue determinada mediante correlación de Spearman en el programa IBM SPSS 19; los datos se graficaron mediante regresión lineal con un intervalo de confianza (IC) del 95\%. El ABC, la Se y Es se determinaron mediante una curva ROC considerando daño hepático el límite superior de referencia de la enzima GDH (>30 U/L) (9-10) o GGT (>39 U/L) (10). Se comparó el ABC entre los analitos utilizando un método de comparaciones para curvas ROC independientes en el programa MedCalc 11.6.1.0 (8). La presentación de daño hepático para cada enzima se contrastó en un tabla de contingencia $2 \times 2$ mediante la prueba de Chi-cuadrado y cuando $p<0.05$ se realizó el análisis de concordancia del índice de Kappa en el programa IBM SPSS 19.

\section{RESULTADOS}

Los resultados obtenidos de las 1566 vacas lecheras se indican en la figura 1 , observándose que la actividad plasmática de las enzimas estudiadas presentó una alta dispersión de datos. Se apreció que el 37, 14 y $30 \%$ de las 1566 vacas tenían valores superiores a los límites de referencia establecidos por el laboratorio para GDH, AST y GGT, respectivamente (10).

La actividad plasmática de AST presentó mayor asociación con la de GDH ( $r=0.55$; Figura $2 a$ ) que la GGT ( $r=0.44$; Figura 3a), así como una mayor $A B C(p=0.01$; Figura $2 b$ y $3 b)$, al utilizar $G D H$ >30 U/L para indicar daño hepático (Tabla 1).
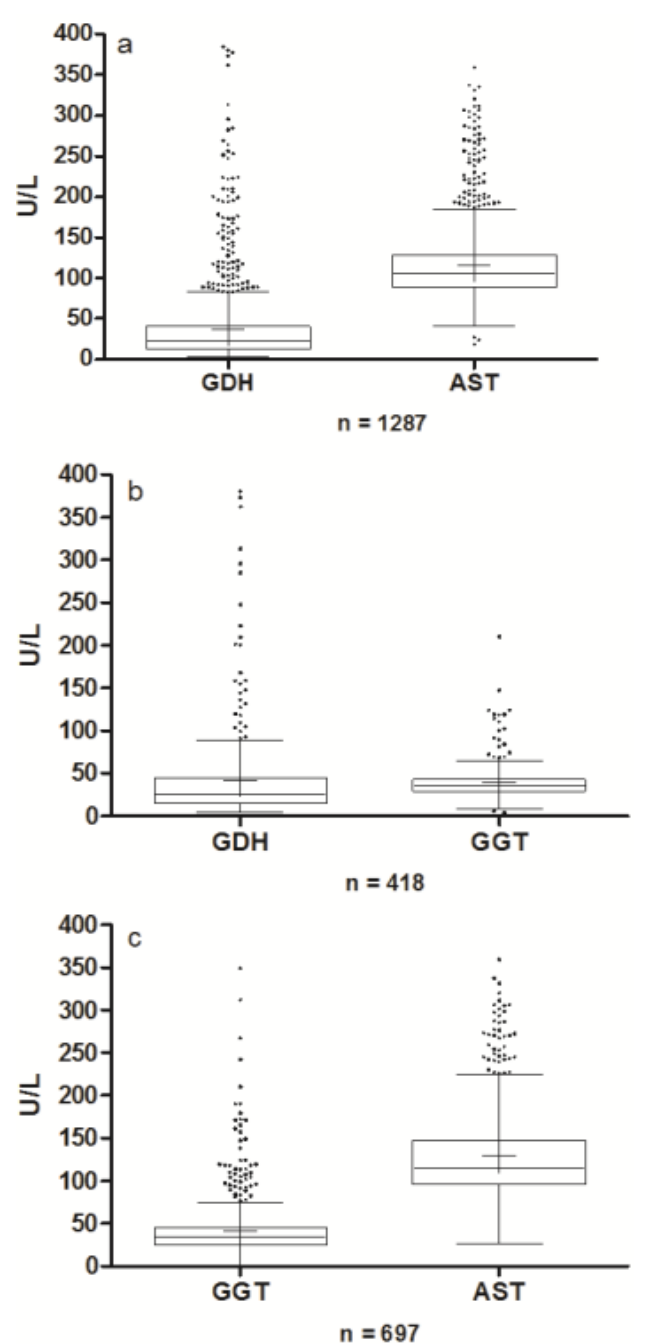

Figura 1. Actividad plasmática de GDH y AST (a), GDH y GGT (b) y GGT y AST (c) en perfiles bioquímicos de vacas lecheras. En el gráfico de caja y bigotes: += media; $\bullet$ indica outliers.

La Se de la AST fue baja (28.2\%), presentando una alta Es $(94.8 \%)$ al utilizar el límite de referencia entregado por el laboratorio como punto de corte (AST<150 U/L) (Tabla 1). La máxima Se y Es de la actividad plasmática de AST para detectar daño hepático en la curva ROC fue cuando su valor alcanzó $>110 \mathrm{U} / \mathrm{L}$, logrando una Se de $70.3 \%$ y un incremento en la concordancia diagnóstica con la GDH ( $>0.300$ en Kappa).

De las 697 vacas con valores de GDH y AST, el 35.6 y el $13.4 \%$, respectivamente, tuvieron la actividad plasmática de dichas enzimas sobre sus valores de referencia. De los animales con GDH $>30 \mathrm{U} / \mathrm{L}$ sólo un $27 \%$ tenían AST $>150 \mathrm{U} / \mathrm{L}$, porcentaje que se eleva al $43.7 \%$ al disminuir el punto de corte de AST a >110 U/L. 

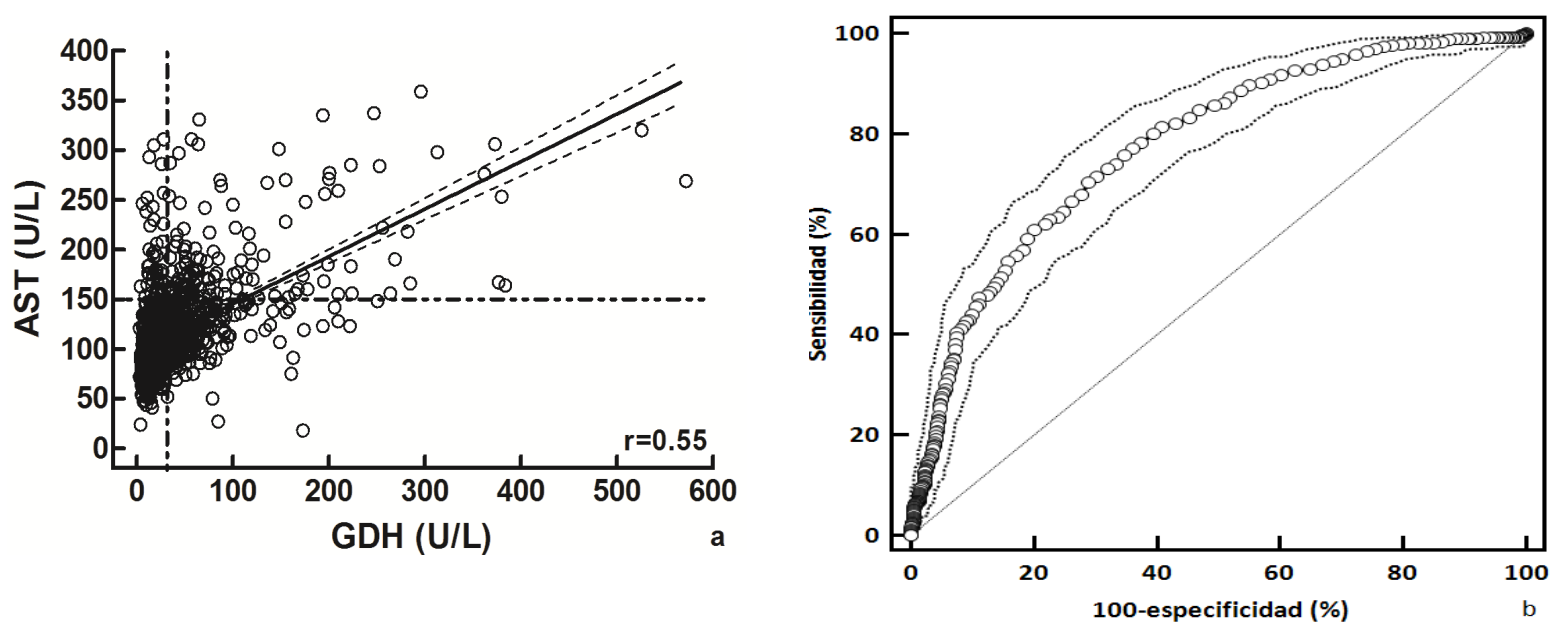

Figura 2. Regresión lineal ( $\pm 95 \%$ IC) entre las actividades plasmáticas de AST y GDH (a) y curva ROC ( $\pm 95 \%$ IC) de la actividad plasmática de AST en vacas lecheras con daño hepático diagnosticado cuando GDH $>30 U / L$ (b). En a, - - - indica límite superior de referencia.
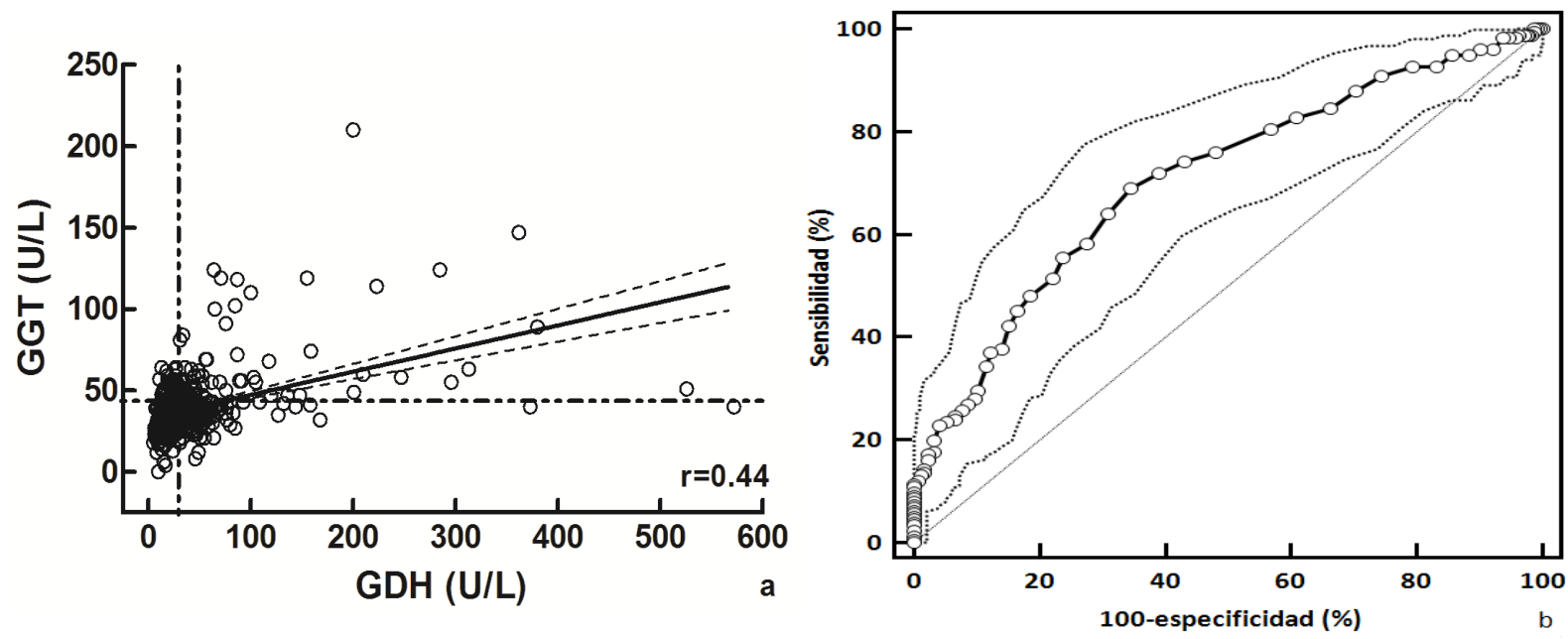

Figura 3. Regresión lineal (IC 95\%) entre la actividad plasmática de GGT y GDH (a) y curva ROC (IC $95 \%$ ) de la actividad plasmática de GGT en vacas lecheras con daño hepático diagnosticado cuando GDH $>30$ U/L (b). En a, - - - - indica límite superior de referencia.

Tabla 1. Área bajo la curva $(A B C)$, sensibilidad (Se), especificidad (Es) y concordancia (Kappa) de la actividad plasmática de GDH, AST y GGT en vacas lecheras con daño hepático diagnosticado cuando GDH > $30 \mathrm{U} / \mathrm{L}$ y GGT $>39 \mathrm{U} / \mathrm{L}$.

\begin{tabular}{cccccc}
\hline & \multicolumn{3}{c}{ GDH >30 U/L } & \multicolumn{2}{c}{ GGT >39 U/L } \\
& AST > 150 & AST >110 & GGT >39 & AST >150 & AST >110 \\
$\mathbf{U} / \mathbf{L} *$ & $\mathbf{U} / \mathbf{L}$ & $\mathbf{U} / \mathbf{L}$ & $\mathbf{U} / \mathbf{L}^{*}$ \\
& $\mathrm{n}=1287$ & $\mathrm{n}=1287$ & $\mathrm{n}=418$ & $\mathrm{n}=697$ & $\mathrm{n}=697$ \\
\hline ABC & 0.778 & 0.779 & 0.708 & 0.714 & 0.714 \\
IC ABC & $0.75-0.80$ & $0.75-0.80$ & $0.66-0.75$ & $0.68-0.75$ & $0.68-0.75$ \\
Se (\%) & 28.2 & 70.3 & 51.4 & 41.0 & 75.7 \\
IC Se & $24.1-32.5$ & $65.9-74.5$ & $43.8-59.0$ & $34.7-47.5$ & $69.8-81.0$ \\
Es (\%) & 94.8 & 71.1 & 77.8 & 85.2 & 52.6 \\
IC Es & $93.1-96.2$ & $67.8-74.1$ & $72.0-82.8$ & $81.6-88.3$ & $47.9-57.3$ \\
Kappa & 0.267 & 0.394 & 0.299 & 0.282 & 0.245 \\
\hline
\end{tabular}

*Punto crítico de máxima sensibilidad y especificidad.
En los perfiles analizados 418 vacas tenían valores de GDH y GGT, entre ellas el 41.9 y $34.4 \%$ presentaron valores de GDH y GGT, respectivamente, sobre el límite superior de referencia. El 50.4\% presentó valores de GGT >39 U/L cuando el de GDH fue > 30 U/L. Además, la GGT presentó una mayor Se y concordancia diagnóstica para detectar daño hepático que la AST >150 U/L e inferior que la AST >110 U/L (Tabla 1).

El grado de asociación entre AST y GGT fue de $r=0.42$ (Figura 4a). AST $>150 \mathrm{U} / \mathrm{L}$ presentó una baja Se para detectar daño hepático. Sin embargo, incrementó su Se al emplear como punto de corte AST $>110 \mathrm{U} / \mathrm{L}$, correspondiente al punto de máxima Se y Es de la curva ROC (Figura 4b), si bien se redujo la concordancia diagnóstica con GGT (Tabla 1). 

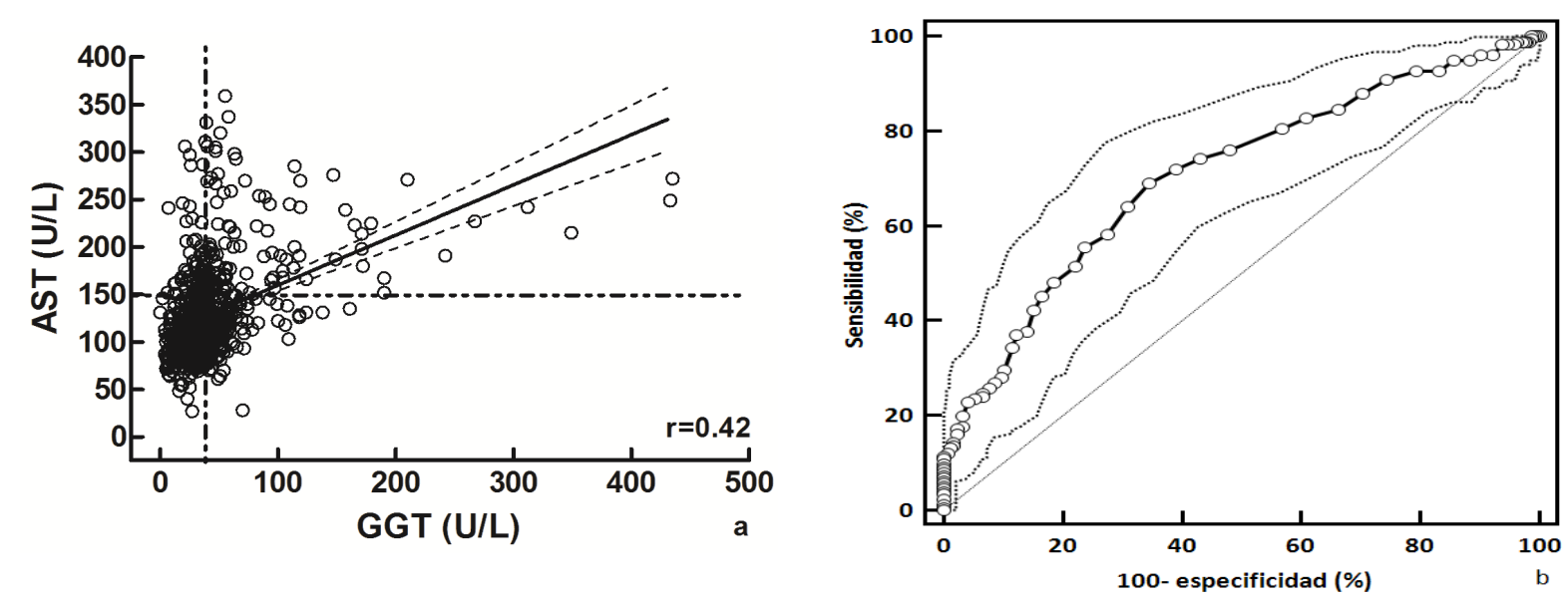

Figura 4. Regresión lineal (IC 95\%) entre las actividades plasmáticas de AST y GGT (a) y curva ROC (IC 95\%) de la actividad plasmática de AST en vacas lecheras con daño canalicular diagnosticado cuando GGT>39 U/L (b). En a, - - - indica límite superior de referencia.

\section{DISCUSIón}

Las actividades plasmáticas aumentadas de las enzimas GDH, AST y GGT observadas en un elevado porcentaje de las vacas indican que estos animales cursaban con algún grado de daño hepático, hepatocelular o canalicular, al momento de obtenerse la muestra para los perfiles bioquímicos (1). La mayor asociación de la AST con la GDH, así como la mayor $A B C$ de la curva ROC para detectar daño hepático, en comparación con la GGT, se atribuye a la ubicación hepatocelular de las dos primeras, no así la GGT que es canalicular $(5,6)$. La GDH plasmática es considerada específica del hígado, motivo por el cual se considera como prueba de oro para el diagnóstico de daño hepático, y por su ubicación mitocondrial, mayormente en la zona 3 del hígado (centrolobular), está incrementada en procesos agudos y tóxicos. Además, el incremento de la actividad plasmática de la GDH se produce con anterioridad al de la GGT, retornando a valores fisiológicos más precozmente por su corta vida media (3), no encontrándose por ello aumentada en procesos crónicos $(4,11)$.

La GGT presenta muy buena Es para detectar daño hepático, incrementando su actividad plasmática por daño canalicular, en casos de colestasis, hiperplasia de ductos biliares, cirrosis y colangiocarcinoma $(1,4-6)$. Por ello, su actividad plasmática está aumentada en vacas con fasciolosis, intoxicación por alcaloides de la pirrolizina y aflatoxinas $(4,12)$.

La AST presentó una baja concordancia diagnóstica con la GDH cuando se empleó como punto de corte el límite superior de referencia utilizado por el laboratorio (>150 U/L) (10).
Resultado atribuible a la inespecíficidad de la AST al estar presente, en sus isoformas citosólica y mitocondrial, en diversos órganos, además de hígado, como eritrocitos, músculos esquelético y cardíaco y limitando su especificidad para diagnosticar alteraciones hepáticas (5). La actividad plasmática de AST aumenta rápidamente, de 6 a 8 horas posterior al daño hepático y habitualmente su incremento es proporcional a la severidad del daño celular, retornando a valores fisiológicos en pocos días. Por ello, su actividad plasmática aumenta de forma marcada en enfermedades agudas, mientras que un aumento leve pudo indicar enfermedades hepáticas crónicas $(4,6)$. La actividad plasmática de AST elevada se observa en vacas con hepatitis infecciosa y tóxica, cirrosis, colestasis y lipidosis hepática; también se observó liberación de AST desde el hepatocito durante estadios de recuperación de injurias hepáticas (6).

El empleo de muestras con hemólisis in vitro u obtenidas de vacas que cursaban con daño muscular explicarían la baja concordancia entre GDH y AST $(5,6)$. Es por eso que frente a la sospecha de una alteración muscular se indica determinar la actividad de la creatina quinasa (CK), la cual está aumentada en daño muscular y no en daño hepático $(5,6)$. A la vez, como la mayor concordancia y punto de máxima Se y Es de la AST en la curva ROC fueron obtenidos con una actividad plasmática de AST mayor a 110 $U / L$, se infiere que el límite superior de referencia de esta enzima debería ser ajustado a este valor.

Las distintas afecciones hepáticas afectan de forma diversa el comportamiento de liberación enzimática. Por ejemplo, en la lipidosis hepática la actividad plasmática de GDH se incrementa más 
intensamente que las de GGT y AST (2). Por otro lado, en cabras intoxicadas experimentalmente con esporodesmina, toxina del hongo Pithomyces chartarum que produce el eczema facial, se observó un incremento de las actividades plasmáticas de la AST y GDH a los 7 días con un pico a los 14 ó 21 días, retornando a sus valores fisiológicos a los 28 y 35 días, respectivamente, mientras que la GGT incrementó desde el día 10 hasta el día 42 con valores máximos al día 28 y 35 (13). Finalmente, la infestación por Fasciola hepática incrementó la actividad plasmática de GDH y AST desde la $3^{a}$ semana posterior a esta, asociado a la migración parasitaria, mientras que la GGT aumentó desde la $8^{a}$ semana, cuando el parásito adulto se ubicó en el canalículo biliar (14).

En conclusión, la actividad plasmática de AST en vacas lecheras se asocia mayormente con la de GDH que con la de GGT y su valor con máxima Se y Es para predecir daño hepático correspondería a $>110 \mathrm{U} / \mathrm{L}$.

\section{Agradecimientos}

A Helga Böhmwald Tecnóloga Medica, por su cooperación en los análisis bioquímicos.

\section{REFERENCIAS}

1. Tennant BC. Hepatic function. In: Clinical Biochemistry of Domestic Animals, Kaneko J], Harvey JW, Bruss ML. San Diego, California: Academic Press; 2008.

2. Bobe G, Young JW, Beitz DC. Invited review: pathology, etiology, prevention, and treatment of fatty liver in dairy cows. J Dairy Sci 2004; 87:3105-3124.

3. Santos JCA, Riet-Correa F, Simões SVD, Barros CSL. Patogênese, sinais clínicos e patologia das doenças causadas por plantas hepatotóxicas em ruminantes e eqüinos no Brasil. Pesq Vet Bras 2008; 28:1-14.

4. Pearson EG. Enfermidades do sistema hepatobiliar. In: Medicina interna de grandes animais, Smith BP. Barueri, SP: Editora Manole; 2006.

5. Hoffmann WE. Diagnostic enzimology of domestic animals. In: Clinical Biochemistry of Domestic Animals, Kaneko JJ, Harvey JW, Bruss ML. San Diego, California: Academic Press; 2008.

6. Stockham SL, Scott MA. Enzymes. In: Fundamentals of Veterinary Clinical Pathology, Stockham SL, Scott MA. Iowa, USA: Blackwell Publising; 2008.

7. Weschenfelder M, Barboza C, Wagemann C, Böhmwald $H$, Chihuailaf $R$, Wittwer $F$ et al. Presentación de desbalances energéticos y alteraciones hepáticas en rebaños lecheros de Chile durante 19862010. XXXV Congreso Sociedad Chilena de Producción Animal. Coyhaique; 2010.
8. Gardner IA, Greiner M. Receiver-operating characteristic curves and likelihood ratios: improvements over traditional methods for the evaluation and application of veterinary clinical pathology tests. Vet Clin Pathol 2006; 35:8-17.

9. Kaneko JJ, Harvey JW, Bruss ML. Clinical Biochemistry of Domestic Animals. San Diego: Academic Press; 2008.

10. Wittwer F. Manual de Patología Clínica Veterinaria. Valdivia: América; 2012.

11. Radostits OM, Gay CC, Blood DC, Hinchcliff KW. Em: Doenças do fígado e pâncreas. Clínica Veterinaria. Um tratado de Doenças dos Bovinos, Ovinos, Suínos, Caprinos e Eqüinos, Radostits OM, Gay CC, Blood DC, Hinchcliff KW. Rio de Janeiro, Brasil: Editora Guananbara Koogan S.A; 2000.

12. Melo MM, Nascimento EF, Oliveira NJF. Intoxicação de bovinos por aflatoxina B1 presente em polpa cítrica: relato de um surto. Arq Bras Med Vet Zoot 1999; 51:555-558.

13. Smith BL, Embling PP. Facial eczema in goats: The toxicity of sporidesmin in goats and its pathology. N Z Vet J 1991; 39:18-22.

14. Benchaoui HA, McKellar QA. Effect of early treatment with rafoxanide on antipyrine clearance in sheep infected with Fasciola hepatica. Xenobiotica 1993; 23:439-448. 